\title{
Liver metastases of colon cancer
}

Ruiz Casado and Pereira in this issue of the "Oncología"l reviews extensively all the new aspects of therapy of metastatic colorectal cancer. A first comment is that it is written by a medical oncologist and a surgeon. This is yet representative of the real multidisciplinary approach that has emerged in recent years in the treatment of metastatic colorectal cancer.

Some years ago, little collaboration existed between oncologists and surgeons. Medical oncologists were in charge of $90 \%$ of patients and treated them with poorly efficient drugs resulting in a short-term palliative approach. Surgeons treated $10 \%$ of patients with a hope of long term survival but with a non neglectable risk of mortality and morbidity... Results have improved both for chemotherapy and surgery, resulting not only into increased survival in both groups but mainly into a synergy of benefits for the patients, that has lead to a dramatic change in the outcome.

The article emphasizes the key-points of this revolution.

Improvement of surgical results is documented with decreasing mortality rates. Achieving hepatic resection is now possible with a perioperative mortality of $0-2 \%$.

\section{Considerable Changes have occurred within the concept of resectablity}

Some years ago, resection was only considered in patients with no more than 3 unilobar metastases, preferably presenting metachronously, resectable with a tumor margin $>1 \mathrm{~cm}$, in the absence of any extrahepatic tumor $^{2} \ldots$ The authors well show that all these contraindications have been progressively abandonned. The modern definition of resectability with curative intent is the ability of the surgeon to clear all measurable disease from the liver providing the future remnant liver (FRL) is between 20-30\% of healthy liver volume at presentation $^{3}$. Nonetheless definition of unresectability could still remains different from one institution to an other in relation to surgical expertise and experience, and to the subjective approach of the risk-to-benefit ratio of hepatic resection for a given patient.

This absence of consensus on the definition of unresectability clearly impacts the wide range of conversion of patients from unresectable to resectable (15-45\%), after downstaging chemotherapy, in various series ${ }^{4-10}$.

\section{Increasing efficacy of chemotherapy}

While «old» chemotherapy using 5-fluorouracil (5-Fu) and leucovorin could achieve a measurable response in only $20 \%$ of patients, the combination of 5-Fu to more efficient drugs such as oxaliplatin or irinotecan has improved response rate to over 50\% and median survival from 8 months with best supportive care to little over 20 months.

As a consequence, patients initially deemed non resectable have been switched to resectable and submittted to «rescue» surgery ${ }^{4,5}$. The long-term results of this approach very close from that of primarily operated patients $^{9}$ is probably one of the major changes occurred in the recent years. Combination of effective chemotherapy with surgery opens a perspective of cure to patients up to now treated only on a palliative setting... In addition, there is little doubt that new biotherapies such as cetuximab and bevacizumab will probably amplify soon the possibility of secondary surgery in patients initially considered as unresectable... 
However, as stressed by the authors, the increasing cost of new drugs should be balanced with the real improvement that they provide to the patients. The authors also mention another tendancy related to the higher efficacy of chemotherapy: the policy to consider chemotherapy as a first-line treatment for all the patients, including those with resectable metastases. Evidence based data do not support this policy and whereas neoaduvant chemotherapy has proved efficacy in patients with multinodular metastases ${ }^{11}$, the gold standard still remains primary liver resection in patients with limited resectable disease. This attitude is still justified by the potential toxicity that systemic chemotherapy may have on the hepatic parenchyma .

Emerging procedures to improve resectability (portal embolization, liver resection combined with radiofrequency, Two-stage hepatectomy) have also emerged recently, adding their effects to that of neoadjuvant chemotherapy for improving resectability.

In summary, the present results of this constantly evolving panorama is that it is no more conceivable that a patient with metastatic colorectal cancer could be managed by a single specialist. The concept of multidisciplinary «oncosurgical» management of these patients has been translated into practice and has become an obligation as recently pointed out by the 2003 French Conference on recommandations for the treatment of colorectal liver metastases ${ }^{12}$. By this way, oncologists and surgeons are able to provide their combined treatment improvements to each patient, for an optimized outcome.

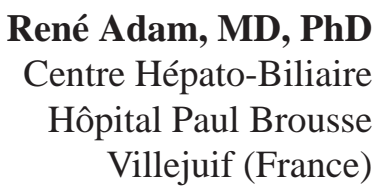

\section{References}

1. Ruiz-Casado A, Pereira F. Liver metastases of colon cancer. New therapeutic approaches. Neoadjuvant chemotherapy. Oncología 2006; 29(1): 3-15.

2. Hughes KS (On behalf of the Registry of Hepatic Metastases). Resection of the liver for colorectal carcinoma metastases: a multiinstitutional study of indications for resection. Surgery 1988; 103: 278-88

3. Poston GJ, Adam R, Alberts S et al. Oncosurge: a strategy for improving resectability with curative intent in metastatic colorectal cancer. J Clin Oncol 2005; 23: 7125-34

4. Bismuth Ann Surg 1996 Bismuth H, Adam R, Levi F, et al: Resection of nonresectable liver metastases from colorectal cancer after neoadjuvant chemotherapy. Ann Surg 1996; 224: 509-20; 520-2.

5. Adam R, Avisar E, Ariche A, et al: Five-year survival following hepatic resection after neoadjuvant therapy for nonresectable colorectal. Ann Surg Oncol 2001; 8: 347-53.

6. Alberts SR, Donohue JH, Mahoney MR et al. Liver resection after 5-fluorouracil, leucovorin and oxaliplatin for patients with metastatic colorectal cancer (MCRC) limited to the liver. A North Central Cancer Treatment Group (NCCTG) phase II study. Proc Am Soc Clin Oncol 2003; 22: 263

7. Zelek L, Bugat R, Cherqui D et al. Multimodal therapy with intravenous biweekly leucovorin, 5-fluorouracil and irinotecan combined with hepatic arterial infusion pirarubicin in non-resectable hepatic metastases from colorectal cancer (a European Association for Research in Oncology trial). Ann Oncol 2003; 14: 1537-1542

8. Pozzo C, Basso M, Cassano A et al. Neoadjuvant treatment of unresectable liver disease with irinotecan and 5-fluorouracil plus folinic acid in colorectal carcinoma patients. Ann Oncol 2004; 15: 933-939

9. Adam R, Delvart V, Pascal G et al. Rescue surgery for unresectable colorectal liver metastases downstaged by chemotherapy: a model to predict long-term survival. Ann Surg 2004; 240: 644-657

10. Rivoire M, de Cian F, Meeus P et al. Combination of neoadjuvant chemotherapy with cryotherapy and surgical resection for the treatment of unresectable liver metastases from colorectal carcinoma. Cancer 2002; 95: 2283-92

11. Tanaka K, Adam R, Shimada H, et al. Role of neoadjuvant chemotherapy in the treatment of multiple colorectal metastases to the liver. Br J Surg. 2003 Aug;90(8):963-9.

12. Chiche L. When is first-line resection of hepatic metastases indicated? Gastroenterol Clin Biol 2003; 27(S2): B11-3, B41-61. 